ACTA UNIVERSITATIS LODZIENSIS

Folia Litteraria Romanica 15, 2020

https://doi.org/10.18778/1505-9065.15.07

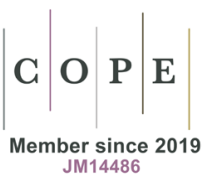

\author{
Jean-Paul Pittion \\ Centre d'Études Supérieures de la Renaissance, Tours \\ Trinity College, Dublin Trinity College of Dublin \\ jean-paul.pittion@univ-tours.fr
}

\title{
L'Adieu du monde, ou Le Mespris de ses vaines grandeurs et plaisirs périssables du Chartreux Dom Polycarpe de la Rivière (1619) : une rhétorique de l'offuscation ?
}

\begin{abstract}
RÉSUMÉ
L'Adieu du Monde ou Le Mespris de ses vaines grandeurs et plaisirs périssables de Dom Polycarpe de la Rivière est paru à Lyon en 1619. Son auteur qui fut chantre de la chapelle de Marguerite de Valois à Usson, et passa neuf ans à la Grande Chartreuse, met en écriture un vaste savoir joignant textes patristiques, érudition humaniste et poésies grecque, latine et française. Dom Polycarpe pratique la rhétorique de la citation, l'assortit de disquisitiones savantes et la met au service d'une dénonciation des attraits du Monde. Il convoque les autorités qu'il cite en juges et témoins. Le discours d'un mépris implacable envers les princes de l'Église, les courtisans et les femmes, n'arrive toutefois pas à masquer la parole d'un homme toujours hanté par la sensualité. Entre amour charnel et amour spirituel, l'Ép $\omega \varsigma$ qui inspire le texte est plus proche de l'ésotérisme de Philon ou de Grégoire de Nysse que de l'á $\gamma \alpha \dot{\pi} \eta \eta$ du maître de la spiritualité cartusienne, Saint Bernard. Le texte de L'Adieu du Monde est le témoin d'une forme de spiritualité partagée par certains cercles humanistes et un exemple d'écriture baroque, au crépuscule de la Renaissance française.
\end{abstract}

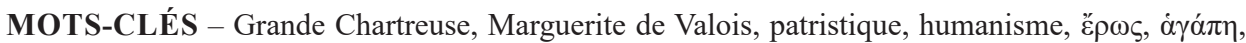
rhétorique, spiritualité, femmes, courtisans, Renaissance, France

"L'Adieu du monde, ou Le Mespris de ses vaines grandeurs et plaisirs périssables by the Cartusian Dom Polycarpe de la Rivière (1619): a Rhetoric of Obfuscation?"

\section{SUMMARY}

L'Adieu du Monde ou Le Mespris de ses vaines grandeurs et plaisirs périssables by Dom Polycarpe de la Rivière was published in Lyon in 1619. The author was chorister in the chapel of the exiled Marguerite de Valois at Usson, and spent nine years in the monastery of la Grande Chartreuse. Dom Polycarpe displays a remarquable knowledge of Patristic and Humanist sources, and a taste for Greek, Latin and French contemporary poetry. The rhetoric of L'Adieu du Monde combines quotations from 
these sources, supported by learned disquisitions, with a relentless denunciation of Church grandees, courtiers and women. Dom Polycarpe summons his authorities as judges and witnesses to his contempt. Nevertheless, his learned rhetoric and his exposé of wordly vanities do not succeed in concealing a sensuality that still haunts him. Between spiritual and sensual love, the ép $\omega \varsigma$ that inspires the text is closer to Philo and Gregory of Nyssa than to Saint Bernard's óyó $\eta$. L'Adieu du Monde is an example of a form of spirituality shared by some humanist circles and of a baroque style of writing practised in the later period of the French Renaissance.

KEYWORDS - Grande Chartreuse, Marguerite de Valois, patrology, humanism, ह̋ $\rho \varsigma$, rhetoric, spirituality, women, courtiers, Renaissance, France

Le thème du mépris du monde est un des thèmes de la poésie à la Renaissance. Dans Le Mespris de la vie et Consolation contre la mort, de Jean-Baptiste Chassignet (Besançon, par Nicolas de Moingesse, 1594), la mort hante la vie et seul le mépris des vanités de la vie conduit à voir dans la mort un réconfort. Dans l'œuvre de Chassignet, on peut voir l'expression littéraire de la Vanitas de l'époque en peinture. Dans le Menosprecio de corte y alabanza de aldea d'Antonio de Guevara (Valladolid, Juan de Villaquirán, 1539), dont plusieurs traductions françaises paraissent à l'époque ${ }^{1}$, la Nature est présentée comme un baume contre le mépris ressenti pour le monde de la Cour. Mépriser le monde, chercher une consolation dans la mort ou l'exil : deux postures face au monde et ses vanités qu'inspire, de façon plus ou moins conscient dans ces œuvres, le courant néo-stoïque de l'époque.

Un autre thème parcourt des écrits de l'époque, celui de l'adieu au monde, première et essentielle étape de l'itinéraire qui conduit l'esprit à Dieu. L'amour du monde est en nous, mais il nous faut lui dire adieu si nous voulons accéder à l'amour qui le transcende, l'amour de Dieu. La source lointaine en est la pensée de Saint Bonaventure, dans des écrits comme la Collatio et contemptu Mundi ou le Stimulus diuini amoris ${ }^{2}$ et les Sermones in Cantica Canticorum de Bernard de Clairvaux ${ }^{3}$. Aux XVI ${ }^{\mathrm{e}}$ et XVII ${ }^{\mathrm{e}}$ siècles, il trouve une nouvelle expression dans les œuvres spirituelles de Louis de Grenade et dans les récits des itinéraires spirituels de Denys le Chartreux et de Benoit de Canfield, qui connaissent une large diffusion.

1 Y compris celle d'E. Dolet, Du Mespris de la court et de la louange de la vie rusticque (Lyon, Estienne Dolet, 1542) et sa réédition, Le Mespris de la court avec la louange de la vie Rusticque. Nouvellement traduict d'Hespagnol en Françoys, Paris, Adam Saulnier, 1543.

2 Nous citons d'après l'édition latine des Euvres complètes de Saint Bonaventure par Charles Peltier. S. R. E. Cardinalis S. Bonauenturae [...]: Opera Omnia Sixti V [...] Jussu Diligentissime Emendata; Accedit Sancti Doctoris Vita, Una Cum Diatriba Historico-chronologico-critica, Parisiis, L. Vivès, 1864-1871, 15 vol. Collatio et contemptu Mundi, t. XII, p. 165 : “Amator enim mundi hujus, quanto amplius Dei obliviscitur, tanto plus a Deo derelinquitur"; Stimulus diuini amoris, t. XII, p. 695 : "ut princeps hujus mundi ejiciatur foras. Tu solus in me regnes, tu solus inhabites mentem meam".

3 S S. Bernardi, Clarae-Valensis abbatis primi, curis D. Joannis Mabillon, presbyteri et monachi benedictini e Congregatione S. Mauri, Parisiis, J.-P. Migne, 1862-1863, 4 vol., Sermones in Cantica Canticorum, vol. IV, p. 780 et sqq. 
La période voit aussi paraître des récits de conversion spirituelle. Il ne s'agit pas de ces récits polémiques qui mettent en scène des conversions entre les deux confessions, conversions réelles ou programmées, parfois forcées ${ }^{4}$, dont on a dénombré plus de 300 ouvrages parus chaque année entre 1598 et $1630^{5}$. Ces textes sont des récits de conversions spirituelles individuelles qui, tout personnels qu'ils soient, se veulent exemplaires. Citons à titre d'exemple L'Adieu du pécheur au péché par le protonotaire $\mathrm{Du}$ Blanc qui connut deux éditions à quelque quarante ans de distance ${ }^{6}$.

Ces deux thèmes du mépris et de l'adieu sont annoncés, joints l'un à l'autre, dans le titre d'un ouvrage du Chartreux Dom Polycarpe de la Rivière ${ }^{7}, L^{\prime}$ Adieu du monde, ou Le mépris de ses vaines grandeurs et plaisirs périssables, publié à Lyon en 1619 par Antoine Pillehotte ${ }^{8}$. L'ouvrage est à première vue déconcertant. En apparence ni son contenu ni son écriture ne le rapprochent d'une œuvre spirituelle. C'est un in-folio de plus de 860 pages. L'encadrement architectural de la page de titre, ornée d'une belle gravure sur bois, annonce plutôt le commentaire savant que l'ouvrage de piété. À consulter la table des matières placée en début de volume, à la suite d'une Épître, d'une Dédicace, d'un «Advertissement au Liseur » et de deux brèves " permissions d'imprimer $»^{9}$, on attendrait un traité de théologie morale ou un sermonnaire destiné à fournir matière à des homélies plutôt qu'un guide spirituel : l'ouvrage est réparti entre quatre livres divisés en chapitres, et traitant successivement « Des Vanités » (liv. I), « De l'Amour » (liv. II), « Des Vertus » (liv. III) et « De la Vie contemplative » (liv. IV). Un prédicateur contemporain aurait reconnu dans ces titres l'écho des thèmes les plus couramment traités dans les homélies des Pères grecs et romains de l'Église ancienne.

4 Voir La Conversion au XVII ${ }^{e}$ siècle. Actes du XII colloque de Marseille, janvier 1982, éd. L. Godard de Donville, Marseille, Centre méridional de rencontres sur le XVII siècle, 1983.

5 Cf. É. Kappler, Les Conférences théologiques entre catholiques et protestants en France au XVII siècle, Paris, Honoré Champion, 2011.

6 Anvers, C. Plantin, 1557 et Arras, J. de La Rivière, 1616.

7 Sur Dom Polycarpe, voir l'article « La Rivière, Dom Polycarpe chartreux, vers 1586-1639 ? ", in Dictionnaire de spiritualité ascétique et mystique, doctrine et histoire, Paris, Beauchesne ; URL : http://beauchesne.immanens.com/appli/article.php?id=12457 ; consulté le 2.11.2018 ; Nouvelle biographie cartusienne, éd. revue, corrigée et augmentée, $2^{\mathrm{e}}$ partie, Grande Chartreuse, 2005, l'article « De la Rivière, Polycarpe », vue 110 ; URL : https://cercornum.univ-st-etienne.fr/ analectacartusiana/sites/default/files/acpdfsbillets/NBC_2-Religieux_chartreux-2005.pdf; consulté le 2.11.2018 ; et É. Daronnat, « Lexique mystique. France - XVII ${ }^{\mathrm{e}}$ siècle », mis à jour en 2014 ; URL : https://grac.univ-lyon2.fr/lexique-mystique-1-r--565754.kjsp ; consulté le 2.11.2018

8 La date figurant sur la page de titre est 1618. Mais la plus récente des pièces liminaires, "L'Epistre » de Dom Polycarpe à Dom Bruno, écrite " de vostre [Chartreuse] de Sainte-Croix en Jarez », est datée du 1er janvier 1619. Les pièces liminaires dont les cahiers étaient signés séparément, étaient imprimées en dernier. On connaît d'ailleurs une variante de la page de titre avec la date 1619 .

9 Elles sont accordées à l'ouvrage par Claude Deville, « député de l'Archevêque de Lyon à l'approbation des livres de son diocèse » et par Thomas Meschatin La Faye, son Vicaire Général. 
Quant au style de l'ouvrage, il n'a rien de la simplicité ou de la réserve de ton auxquelles on pourrait s'attendre dans un ouvrage de spiritualité. Dans cet «Advertissement » qui précède la table des matières, Dom Polycarpe prévient le lecteur " de ne pas demander de ces belles fleurs qui naissent sous le doux air de la Cour ", mais, il y déclare aussi, de façon candide, que l'ouvrage est un divertissement qu'il s'est « amuzé à façonner, y mettant en œuvre mille beautés de l'Antiquité, mille rares inventions des poëtes et orateurs, rehaussées des hautes et divines sentences des Saints Pères $»^{10}$.

Notre étude cherchera à montrer que $L^{\prime}$ Adieu du Monde, en dépit des premières apparences, n'est pas qu'une volumineuse compilation d'extraits destinés à fournir matière à un prédicateur en mal d'inspiration pour son sermon du jour ou des fleurs de rhétorique pour l'orner. Dans son « Epistre liminaire » à Dom Bruno d'Affingues, Général de l'ordre des Chartreux et Prieur de La Grande Chartreuse, Dom Polycarpe rappelle humblement la conversion qui est à l'origine du livre. Il adresse au Prieur, écrit-il, « ces premières pensées de [sa] conversion, ces premiers efforts d'un esprit encore tout duvet $»$. Le thème qui parcourt le livre et qui lui confère une dimension proprement spirituelle, est celui de la fausseté de l'amour humain et de l'irrésistible l'élan de l'âme vers Dieu. L'œuvre est l'expression à peine avouée d'un combat intérieur de plusieurs années, qui a conduit celui qui l'a vécu à la conversion.

Dans L'Adieu du Monde, Dom Polycarpe se présente, en effet, sous un double visage. Il est le « je », locuteur du texte, qui guide le lecteur par de multiples interventions dans son discours : « voyez », « remarquez », « je l'ay dict et je le redis »; ou encore « certes je ne me trompe pas »; ou bien même (p. 566) «ne m'arrestant sur icelui [ce point] icy davantage, puisque j'ay creusé la veine en la fouillant davantage ». Mais un autre « je» est le sujet de son propre texte et c'est lui aussi qui s'exprime par la parole des autorités qu'il convoque. Le «nous » par lequel il les fait parler, est aussi bien le sien que le nôtre. Et lorsqu'elles s'adressent à « vous », c'est à nous qu'elles s'adressent, nous lecteurs encore captifs du monde, et exhortés à le mépriser et à lui dire adieu.

Cette manière de façonner le texte par le truchement des auteurs que Dom Polycarpe cite, traduit et paraphrase, crée un effet d'oralité. Le ton des citations des Pères est solennel. Il est celui de la prédication, de l'admonition et de l'objurgation. À leur voix Dom Polycarpe joint celles de nombreuses autorités de l'Antiquité grecque et romaine. La concision des apophtegmes qui viennent en point d'orgue, donne d'autant plus de force à l'exhortation. Ce n'est pas un hasard si, dans son " Advertissement », Dom Polycarpe s'adresse au « liseur » plutôt qu' au « lecteur », terme de loin le plus fréquemment employé dans les discours préfaciers de l'époque. Selon Jean Nicot, dans le Thrésor de la langue françoyse, le « liseur » est celui qui

${ }^{10}$ Lorsqu'elles sont incluses dans notre texte, nous respectons la présentation originale des références en les plaçant entre parenthèses et guillemets, en modernisant la ponctuation et en développant les titres, si nécessaire, à l'aide de [...]. 
lit à quelqu'un qui l'écoute, mot équivalent du mot latin praelector qui désignait celui qui fait la lecture aux enfants.

Dom Polycarpe le reconnaît dans l'«Advertissement », il n'a pu s'empêcher de se laisser aller au plaisir de discourir. « Ainsi ay-je laissé aller ma plume au penchant et fil de mes conceptions ; non toutefois si indiscrètement que j'aye oublié le tissu de mon ouvrage, et que la variété, en laquelle se plaisent les hommes de ce siècle ne leur fasse ces destours agréables $\gg\left(\mathrm{f}^{0} * * 3 \mathrm{v}^{\circ}\right)$. Dans le texte, Dom Polycarpe cultive la uarietas et, par amplification, la citation conduit parfois, de glissement en glissement, à des développements substantiels. Par exemple, une séquence textuelle consacrée à un elogium de l'amitié occupe les pages 376 à 383 du chapitre 13 du livre II. L'elogium débute par une série de définitions de l'amitié fidèle, empruntées au chapitre 6 de l'Ecclésiastique, adaptées en français dans le corps du texte et, de plus, rapportées à la marge selon la version latine de la Vulgate : «L'Amy fidele [...] est une forte protection ». L'idée de comparer l'amitié et l'amour, soutenue, entre autres, par une référence à l'aide mutuelle entre un aveugle et un claudicant ( $\mathrm{p}$. $378^{11}$ ), débouche sur l'image de l'âme embrasée par le feu précédant une paraphrase des Dialogues 50 et 52 de Pétrarque, accompagnée en manchette d'une longue citation latine. Le développement prend appui ensuite sur des citations de Cicéron (De amicitia) et du jésuite espagnol, Martin de Roa (in suis singularibus libris) ${ }^{12}$, pour rebondir sur plusieurs emprunts à Pline, tirés du livre 2, chapitre 41 et du livre 25 , chapitre 8 . Ces citations ont trait à la fleur tournée vers le soleil et à l'hirondelle retrouvant son nid, avec toute une suite d'exemples botaniques - qui sont autant de figures de la fidélité. D'un intertexte à l'autre, l'écriture de Dom Polycarpe s'enrichit de sens nouveaux et approfondit la matière de son traité.

Si la chaîne du «tissu » du livre est faite des « beautés » de l'Antiquité, des « inventions » des poètes et orateurs, et des « sentences » des Pères, sa trame par contre, est formée d'une argumentation consistant en une suite de preuves et contre-preuves qui visent à faire justice de la défense du Monde, de ses attraits et ses grandeurs. Cette façon d'argumenter est caractéristique des plaidoyers écrits de l'époque, que le Thresor de la langue francoyse définit comme une altercatio de admittendis aut rejiciendis testimoniis. Un lecteur moderne risque parfois de s'y perdre, mais une telle façon de procéder était familière aux lecteurs de l'époque, connaisseurs de la rhétorique judiciaire. Notons, à ce propos, que l'ouvrage est dédicacé à Balthazar de Villars ${ }^{13}$, conseiller du Roi et Président au Présidial

${ }^{11}$ Cf. Alciat, Emblemata Andreae Alciati I. C. clariss. Latinogallica / Les Emblemes latin-françois du seigneur André Alciat excellent jurisconsulte, trad. Cl. Mignault, Paris, Jean Richer, 1584, $\mathrm{f}^{\mathrm{0}} 221 \mathrm{r}^{\mathrm{0}}-\mathrm{v}^{\mathrm{0}}$ : emblema CLX Mutuum auxilium.

${ }^{12}$ Martin de Roa S. J, jésuite espagnol (1561-1637). Il existe plusieurs éditions contemporaines de l'ouvrage dont l'une lyonnaise : M. de Roa, Singularium locorum ac rerum libri V, Lugduni, sumptibus Horatii Cardon, 1604.

${ }^{13}$ L'Armorial historique de Bresse, Bugey, Dombes, Pays de Gex, Valromey et Franc-Lyonnais, édition du Révérend Édmond Du Mesnil, Lyon, 1872, p. 693, fournit des renseignements sur les de Villars. 
de Lyon ${ }^{14}$. Réfuter point par point les affirmations de la partie adverse - dans ce cas-ci que le Monde a ses plaisirs et ses attraits -, rappelle aussi la méthode de défense souvent adoptée par les apologistes de l'Église des Premiers Temps, en particulier Tertullien, très présent et cité près de cinquante fois dans L'Adieu $d u$ Monde, notamment son Apologétique (par exemple, p. 347, 464 et 608).

Elocutio du prédicateur, dispositio du plaideur, et aussi doctum ingenium du lettré. Car citer fournit à Dom Polycarpe l'occasion de disserter. Au livre III, pour prendre un parmi de nombreux exemples, après avoir discuté du sens de l'idée de « gouverneur», invectivé les courtisans et dénoncé ceux qui « ont suyvy le train de Caïn et se sont desbordés en l'erreur du loyer de Balaam »- les protestants qui ne reconnaissent pas l'autorité du Pape - il se lance, aux p. 444-446, dans une longue disquisitio sur le sens du verbe « paître » qui, selon lui, signifie « gouverner ». Il fait alors appel au chapitre 21 de l'Évangile de Jean, vraisemblablement le verset 1 , où Jésus dit à Simon : " paix à mes agneaux ». Il renvoie au chapitre 44 d'Isaïe où le verbe, nous le citons, « ne signifie pas seulement paistre, mais régir et gouverner». Puis Dom Polycarpe cite en grec l'Iliade (chant 2, v. 85), l'épisode où Nestor

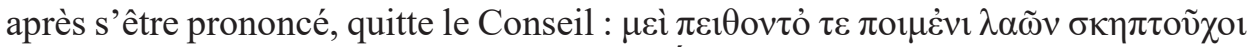
$\beta \alpha \sigma i \lambda \tilde{\eta} \varepsilon \zeta \dot{\varepsilon} \pi \varepsilon \sigma \sigma \varepsilon \delta ̉ v \tau o ~ \delta \varepsilon \lambda \alpha o i$, ainsi que l'Évangile de Matthieu (chap. 2, v. 6), en citant en manchette la Vulgate, "Ex te erit mihi dux qui regat populum meum Israël". Dom Polycarpe conclut sa dissertation en rappelant que Matthieu cite ici Michée (chap. 5, v. 2) et précise en caractères hébraïques qu'il y a deux lectures possibles de ce verset : non « rohhi, qui signifie paistre; mais moschel, qui veut dire gouverner et régir $»^{15}$.

Les disquisitiones qui fleurissent dans la « fantasque bordure et brodure des marges » («Advertissement » $\mathrm{f}^{\circ} * * 4 \mathrm{v}^{\circ}$ ), rappellent les échanges savants qui animaient les cénacles de lettrés et leurs conversations érudites où chacun s'envoyait la balle. Une liste sommaire des principaux auteurs qui sont cités dans

Balthazar, alors Président au Parlement de Dombes, avait épousé la fille du juriste Nicolas de Lange qui jusqu'à son décès en 1606 réunit dans sa résidence de l'Angélique, un cénacle de doctes auquel on attribue ce nom en 1606. Balthazar décéda en 1629.

${ }^{14}$ La sénéchaussée de Lyon a été érigée en présidial lors de la création des présidiaux par Henri II. $C f$. Archives Départementales du Rhône, «Fonds de la sénéchaussée et du siège présidial de Lyon ». Sur Lyon à la Renaissance, voir Intellectual Life in Renaissance Lyon. Proceedings of the Cambridge Lyon colloquium, 14-16 April 1991, ed. by Ph. Ford, G. Jondorf, Cambridge, Cambridge French Colloquia, 1993 ; voir aussi Actes du Colloque sur l'humanisme lyonnais au XVI' siècle. Mai 1972, Grenoble, Presses Universitaires de Grenoble, 1974 ; Il Rinascimento a Lione. Atti del Congresso internazionale, Macerata, 6-11 maggio 1985 a cura di Antonio Possenti, Giulia Mastrangel, Roma, Pubblicazioni della Facoltà di lettere e filosofia, Università di Macerata, 1988.

${ }^{15}$ Le mot rohhi est une translittération approximative du mot hébreu רע qui signifie " berger ».

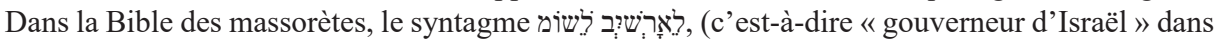
la version Chouraki) se trouve au verset 1 et non 2 du chapitre 5. Le numéro 2 lui est attribué dans les versions chrétiennes. L'importance du verset pour l'hermétisme chrétien de la Renaissance tient à ce que pour Rashi et le Targum Jonathan, le verset est l'annonce de la venue du Messie. 
le chapitre 1 du livre I donnera un avant-goût de l'érudition de Dom Polycarpe. On y rencontre, entre bien d'autres, des citations de Pères de l'Église, comme Ambroise, Isidore, Tertullien, mais aussi les Cappadociens, Grégoire de Nysse, Grégoire de Nazianze et Basile de Césarée; des citations d'écrivains latins (Ovide, Salluste, Sénèque, Virgile, Ausone et Lucain). On y trouve aussi des emprunts aux textes de philosophes anciens ou de leurs commentateurs (Aristote, Boèce, Épictète, Macrobe, Olympiodore, Platon), d'historiens de l'Antiquité (Élien le Sophiste, Valère Maxime), ainsi que des citations d'œuvres d'humanistes et de lettrés de l'époque (notamment Jules César Scaliger et Pontus de Tyard).

On rencontre aussi, dans L'Adieu du Monde, des citations de poètes français de l'époque qui émaillent le texte : un octonaire de La Roche Chandieu : «L'eau va vite en s'escoulant / Plus vite le traict volant » (p. 480 $)^{16}$; un autre de Ronsard, De la vanité du monde : "Soudain comme on voit la fleur / Sans sa première couleur » (p. 392). Dom Polycarpe cite aussi entre bien d'autres des vers « d'un autre François non moins délicat », « l'Ovide de France » (Philippe Desportes), tirés du recueil Le Premier livre des Amours de Diane : « Les plus lourds animaux parmi les gras herbages / Sentans cet aiguillon, qui leur poinct les courages, / Bondissent furieus, pleins d'amoureux désir» (p. 353), et « Durant le grand débat de la Masse première » (p. 354) ${ }^{17}$.

Au-delà des occasions qu'elle offre à Dom Polycarpe de partager avec son « liseur » son goût pour les poètes à la mode, et de déployer son érudition, sa riche culture nourrie de lectures théologiques, savantes et profanes est mise au service d'un combat intérieur que l'écriture de L'Adieu du Monde laisse entrevoir. "Imaginez-vous ainsi, écrit-il, que ces âmes bienheureuses, au commencement de leur conversion se voient aucunement investis [sic] des vagues et des flots, assaillis de la tormente » (p. 764). S'y trouve intercalée une citation latine Diabolus enim semper primordia, tentat rudimenta uirtutum, sancta in ipso ortu festinat extinguere, sciens quod ea euellere fundata non possit avec en note la référence à Petr[us] Chrysolog[us] homil[ia] II. Toutefois, l'image de la «tormente » de l'âme surgit, malgré lui, à plusieurs reprises ${ }^{18}$. L'ouragan de citations que déchaîne l'écriture de Polycarpe est la manifestation de la tempête intérieure qui a agité et agite encore son âme. "Quel est le navire, s'exclame-t-il, qui ne varie sur les ondes instables de la mer? Quelle est la voile laquelle ne s'enfle quand elle a le vent en pouppe, qui me donnera un homme ferme comme un rocher parmi les divers branles de l'ondoyante marée des vanités ?» (p. 433-434). Suit ici aussi une citation

${ }^{16}$ A. de La Roche Chandieu, Méditations sur le psalme XXXII. Traduictes de Latin en François et reveues par l'auteur mesme. Avec une preface à ceux qui se sont despartis de l'Eglise reformee. Ont été adjoustez cinquante octonaires sur la vanité du monde, par A. Zamariel, Genève, G. Laimarie, 1583.

${ }_{17} \mathrm{Ph}$. Desportes, Les Premieres $2 e u v r e s$ de Philippes Des Portes, Au Roy de Pologne, Paris, Robert Estienne, 1573, p. 24-25 : «Chant d'amour», v. 50-56.

${ }_{18}$ Par exemple, p. 610, dans une traduction qu'il donne d'un passage de l'Épître 9 de Saint Cyprien. 
latine composite tirée celle-ci de l'Agamemnon de Sénèque le jeune (v. 64-65 et v. 71-76) : Non sic Libycis syrtibus aequor / Furit, alternos uoluere fluctus, etc.

Un seul passage de l'ouvrage fait entrevoir le contrecoup qu'a pu avoir l'arrivée au Désert de la Grande Chartreuse, difficilement accessible à l'époque ${ }^{19}$, sur un Polycarpe de la Rivière qui avait passé ses jeunes années à la cour de Marguerite de Valois. Il écrit à l'adresse des « petits aiglons » qui aspirent à suivre son exemple, " si l'horreur des déserts, les pentes des rochers et le son résonnant des furieux torrents emporte dès la porte de vostre conversion l'ardeur de vos courages, pensez que la résolution que vous avez prise de vivre separé du Monde, vous porte heureusement à une vie qui n'a point de mort» (liv. IV, chap. 4, p. 722) ${ }^{20}$.

«Il y a neuf ans maintenant », écrit-t-il dans l'« Advertissement », « que [...] Dieu me donna la volonté, et quant et quant le moyen de sortir du service d'une grande Princesse, pour me donner au sien en cete reigle » $\left(\mathrm{f}^{\mathrm{0} * *} 2 \mathrm{r}^{\circ}\right)$. Entré comme jeune choriste, probablement en 1595, au service de Marguerite de Valois, alors exilée à Usson, François de la Rivière le quitta pour entrer au monastère de la Grande Chartreuse en 1609, six ans avant le décès de Marguerite ${ }^{21}$. L'expression « quant et quant », 'à la longue', déjà un peu vieillie, qu'il utilise dans l' "Advertissement », suggère qu'il a hésité un certain temps avant de décider d'entrer en religion. L'âge de vingt et un ans était l'âge minimum requis par l'ordre, pour prendre l'habit de novice.

Dom Polycarpe sortit de la Grande Chartreuse sept ans plus tard en 1616, lorsqu'il fut choisi par l'ordre pour prendre la fonction de procureur de la chartreuse du Lys-Saint-Esprit, établie depuis 1584 sur la colline de la Croix-Rousse de Lyon ${ }^{22}$. Des trois années qu'il passa à Lyon datent ses deux premiers ouvrages, les Récréations Spirituelles, paru, sans nom d'auteur, au début de l'année $1617^{23}$, et L'Adieu du Monde, paru au début de l'année 1619. Cette année-là, il quitta Lyon, lorsqu'il fut nommé prieur de la Chartreuse de Sainte-Croix-en-Jarez.

La conversion est déjà présente dans les Récréations Spirituelles. Ce premier ouvrage consiste en une suite de dialogues entre plusieurs protagonistes. Au livre III,

${ }^{19}$ Deux chemins escarpés conduisaient au val de la Grande Chartreuse, au nord par le col de la Ruchère, au sud par les gorges du Guiers, puis par l'alpe de Bovinant.

${ }^{20}$ Jamais en manque de citations, Dom Polycarpe cite ici quatre vers latins tirés de la Phaedra de Sénèque le Jeune (monologue d'Hyppolite, v. 482 et suiv.).

${ }^{21}$ Sur le monastère à l'époque, on consultera Pépy, Émilie-Anne, Annecy, Académie salésienne, 2013. Les statuts de l'Ordre, rédigés par Guigo de Castro (1083-1013) on fait l'objet d'une édition à Basle, chez Joannes Petri et Joannes Froben en 1610.

${ }^{22} C f$. Lettres patentes d'Henri IV confirmant cette fondation, A. D. Rhône, « Fonds Coste » MS 265. Le Procureur d'une maison religieuse était son économe chargé des relations avec le monde extérieur.

${ }^{23}$ [Dom Polycarpe de la Rivière], Recreations Spirituelles sur l'amour divin et le bien des amis. Enrichies d'une infinité d'Inventions tres-subtiles et utiles à la Conversion des grandes Ames de la Cour. Tirées de la Bibliotheque de Mre Baltazar de Villars, Conseiller du Roy en son Conseil d'Estat, President en la Seneschaulcée et Siege Presidial de Lyon et premier President au Parlement de Dombes, Paris, chez Regnauld Chaudiere, 1617. 
le chapitre 5 met en scène la conversion de l'un de ces personnages, Dorothée, qui a choisi d'entrer au cloître. Le chapitre inclut, pages 216-218, une lettre de celleci qui exprime bien le sens de semblables conversions : « je m'en vois me perdre pour vous trouver, et mourir en nostre Amour mortel, pour vous faire revivre en l'Amour immortel » (p. 216) ${ }^{24}$. Cette déclaration du personnage de Dorothée est un écho direct d'un thème majeur de la théologie chrétienne, l'opposition entre amour

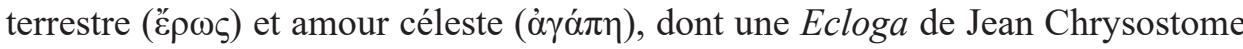
constitue une des expressions les plus connues ${ }^{25}$.

Une autre source, philosophique celle-ci, sur l'amour est le $\Sigma v \mu \pi$ óøıv, le Banquet de Platon. Dans le Banquet, amour charnel et amour spirituel ne s'opposent pas. Ils se distinguent mais se complètent. Dans l'Adieu au Monde, le choix qui se présente à l'homme entre les deux formes de l'amour est exprimé en termes platonisants et dans la façon dont Marsile Ficin interprète Platon plutôt que celle de Pic de la Mirandole ${ }^{26}$, deux interprétations qui ont contribué aux courants néo-platonisants de la Renaissance. L'image néo-platonisante des deux Venus revient à plusieurs reprises dans le texte de Dom Polycarpe (notamment p. 193 et 280).

Les deux ouvrages de Dom Polycarpe se distinguent aussi par leur écriture. Dans L'Adieu du Monde, comparé aux Récréations Spirituelles, le développement des textes-sources est d'une tout autre ampleur. La diversité des œuvres qu'il a lues et utilisées, et qu'il cite, paraphrase et commente, pose la question de savoir où et sous quelle forme il a pu y avoir accès. Faute d'espace, une analyse détaillée des sources que Dom Polycarpe a consultées ne peut être présentée ici. Nous nous contenterons de résumer les conclusions d'une analyse que nous avons par ailleurs conduite parallèlement à celle-ci.

Il est certain que, dans la majorité des cas, Dom Polycarpe a eu une connaissance directe des textes dont il tire parti. Certes, des notes en manchette montrent qu'il a aussi utilisé parfois des recueils imprimés de loci communes ou d'extracta pour y puiser à l'occasion. Il s'est aussi servi abondamment des Exercitationes ${ }^{27}$ de Jules César Scaliger (1484-1558). L'objectif déclaré de Scaliger était de détailler

${ }^{24}$ À réception de cette lettre, Hoplée décide de vendre ses biens et de se faire capucin. Mais il rend une ultime visite à Dorothée dans son cloître, p. 263 et suiv.

${ }^{25}$ Eclogae ex diuersis homilis, Homelia I: "Sed obscœna uoluptas est in amando. Apage et male loqui desine, O Homo: nihil enim ab ejusmodi uoluptate tam immune est quam uera caritas" ; J. P. Migne, Patrologia Graeca, t. LXIII, col, 578. Sur l'histoire de la notion d'amour dans la théologie chrétienne, une référence essentielle reste l'ouvrage d'A. Nygren, Erôs et Agapè, trad. P. Jundt, Paris, Aubier, 1944-1952, 3 vol.

${ }^{26}$ Voir l'excellente mise au point d'É. F. Meylan, «L'évolution de la notion d'amour platonique », Humanisme et Renaissance, 1938, t. 5, no 3, p. 418-442.

${ }^{27}$ Julii Caesaris Scaligeri Exotericarum exercitationum liber quintus decimus, de subtilitate, ad Hieronymum Cardanum, Lutetiæ, Michaelis Vascosiani, 1557. Il existe au moins une autre édition qu'a pu utiliser Dom Polycarpe, celle d'Andrea Wechel (Francfort,1582). 
et de corriger les erreurs commises par Jérôme Cardan dans son De Subtilitate (1550), mais dès sa parution, l'ouvrage a été utilisé comme une sorte de compendium fournissant de multiples références dans ses articles qui couvrent tous les champs de l'érudition humaniste.

Il est clair toutefois que la plupart du temps Dom Polycarpe a procédé en transcrivant dans ses propres recueils de notes, les passages qui l'intéressaient dans les œuvres qu'il consultait. À l'occasion, lorsque le passage en question était trop long ou trop complexe, il en résumait la teneur mais toujours en veillant à conserver la citation ou la référence qui lui paraissait essentielle. Il transférait ces notes dans des registres où il est probable qu'elles étaient rangées sous une suite de mots clés. C'est la méthode des lieux communs, caractéristique de l'humanisme renaissant ${ }^{28}$. On en trouve un exemple dans le célèbre Dialogue de Juan Luis Vivès, dans lequel un maître s'adresse ainsi à son disciple : « apporte-moi mon livre où j'écris ce que je collige et mes registres plus grandes $[\mathrm{sic}] \aleph^{29}$. Seule cette façon de procéder explique, dans l'Adieu du Monde, l'enchaînement de citations qui développent et illustrent une idée (comme dans le premier exemple envisagé ci-dessus) ainsi que les multiples références croisées qui élucident un point particulier (comme c'est le cas dans le second).

D'une analyse détaillée des sources que nous avons effectuée il ressort qu'il est impossible, étant donné la multiplicité et la diversité des ouvrages dont il a tiré des notes, que Polycarpe ait pu entreprendre et terminer ses recherches livresques et composer successivement deux ouvrages durant une période de moins de trois ans, alors qu'il occupait ses fonctions à la chartreuse de la Croix-Rousse. L'Adieu $d u$ Monde est l'aboutissement d'une longue recherche au cours de laquelle il a eu accès à une multiplicité d'ouvrages au contenu divers. Il a pu consulter des éditions des auteurs anciens et se familiariser avec la pensée ésotérique grâce aux ouvrages de la bibliothèque de Marguerite de Valois ${ }^{30}$. La bibliothèque de la Grande Chartreuse contenait - en manuscrits ou imprimée - de nombreuses éditions des

${ }^{28}$ Voir A. Moss, Les Recueils de lieux communs, méthode pour apprendre à penser à la Renaissance, traduit de l'anglais sous la dir. de P. Eichel-Lojkine, Genève, Droz, 2002 ; Voir aussi, A. Blair, "Humanist Methods in Natural Philosophy: The Commonplace Book", Journal of the History of Ideas, 53(4), 541-551 et du même auteur, "The Rise of Note-Taking in Early Modern Europe", Intellectual History Review, Sept. 2010, 20 (3), p. 303-316.

29 Les Dialogues de Jean Loys Vives, traduits du latin en françoys [...] par G. de Housteville, Anvers, G. Cuzman, 1578, sig. I 5-I 6.

${ }^{30} \mathrm{Il}$ existe plusieurs listes partielles d'ouvrages ayant appartenu à Marguerite de Valois, dont une tardive de 1608. Elles ont fait l'objet de plusieurs études, parmi lesquelles, de J.-H. Mariéjol, La Vie de Marguerite de Valois : reine de Navarre et de France : 1553-1615, Paris, Hachette, 1928 (hostile à Marguerite) et principalement d'É. Viennot, Marguerite de Valois histoire d'une femme, histoire d'un mythe, Paris, Payot, 1994 et É. Viennot, Marguerite de Valois : " la reine Margot », Paris, Perrin, 2005. Voir aussi les Actes du Colloque de Nérac, De Marguerite de Valois à la reine Margot autrice, mécène, inspiratrice, sous la dir. de C. Magnien, É. Viennot, Rennes, Presses Universitaires de Rennes, 2019. 
Pères de l'Église et des spirituels de l'Ordre ${ }^{31}$. Comme le précise le chapitre XVII des Statuts cartusiens, le chartreux pouvait emprunter des livres pour les lire en solitaire dans sa cellule ${ }^{32}$. C'est sans doute au monastère que Dom Polycarpe a débuté la rédaction de son ouvrage. Écrire la vérité, disent les statuts de Guigo, trouve sa récompense dans la miséricorde divine accordée aux affligés ${ }^{33}$. Il a complété ses lectures et conclu son texte en consultant la bibliothèque du haut magistrat lyonnais nommé Balthazar de Villars qui a encouragé la publication de ses deux premiers ouvrages ${ }^{34}$.

Les lieux où Dom Polycarpe a eu accès à ses sources sont divers, mais l'orientation qui a guidé ses multiples lectures est restée tout du long, la même. À première vue, l'art de la uarietas et le rôle joué par la citation dans la composition de L'Adieu du Monde évoquent l'écriture du Montaigne des premiers essais. En réalité, les lectures de Dom Polycarpe ne sont pas des lectures «en liberté ». Elles ont un caractère systématique, quasiment obsessionnel. Elles sont inspirées par une recherche sans relâche pour trouver les mots qui puissent adéquatement rendre compte du combat intérieur qui l'a conduit à sa conversion.

Dire adieu à l'amour terrestre pour l'amour céleste, ne veut pas dire qu'on le méprise, comme le montre le passage des Récréations Spirituelles cité plus haut. Le mépris que lui témoigne Dom Polycarpe n'est en réalité que le symptôme d'un combat intérieur où au contraire s'affrontent l'appel de l'amour charnel et celui de l'amour de Dieu. Certes, les trois figures du Monde que Dom Polycarpe couvre de son mépris - le clergé, les courtisans et les femmes - sont les cibles conventionnelles de la satire de l'époque. De ces trois figures, celle du courtisan, le sarcasme avec lequel il la traite, reste proche de la satire traditionnelle : «Ô le galant homme [...] marjolet bien vestu et bien suyvy [...] Ce ne seront que révérences à sa fraize, à sa cappe et à ses bottes. Mais tâtez le pouls à ce pèlerin, vous le rencontrerez un veau revestu, un esprit plat, qui n'aura rien de relevé que les moustaches, bandé sur une démarche estudiée, baguenaudant sans cesse » (liv. III, chap. 6, p. 490).

Par contre le ton qu'il utilise lorsqu'il vitupère contre les clercs et surtout contre les femmes est sans commune mesure avec l'exagération ou la diminution comiques,

${ }^{31}$ Une liste incomplète datant d'environ 1600, le Repertorium librorum domus Cartusie est conservée à la bibliothèque municipale de Grenoble (anctY 107 (1243) (20), cote Y.107 Rés.). Cf. P. Fournier, Notice sur la bibliothèque de la Grande Chartreuse au Moyen Âge. Suivie d'un Catalogue de cette bibliothèque au XVE siècle, Grenoble, Allier, 1888.

32 "Ad cellam semper recurrit, [monachus] ut legendo, orando, meditando et turbulentos animi motus, ex rerum exteriorum cura, uel dispostione surgentes sedare".

33 "Quot enim libros scribimus tot nobis ueritatem [...] facere uidemur, sperantes a diuino mercedes" (Cap. XXVIII).

${ }^{34}$ Selon L'Armorial historique cité note 13, dans son testament de 1624, Balthazar de Villars, légua les livres de son cabinet de curiosités à ses filles et à ses gendres. Un petit nombre d'ouvrages reliés à ses armes, ont plus tard rejoint la Bibliothèque municipale de Lyon. 
procédés traditionnels de la satire. Ses vitupérations tiennent de l'anatomie morbide : « [Voyez], ce moine et ce prestre [dont] à leur ramage vous diriez qu'ils sont descendus du ciel [...]», écrit-il au sujet des religieux. «Voyez-les au dedans, ô que d'hideuses imaginations, ô que de vices énormes et villains ! - Ainsi le Cygne, un si bel animal, si blanc, si net, si poly, si agréable en son chant, [...] est tout sale au-dessous, et a la chair extrêmement noire, dure et mauvaise (liv. III, chap. 6, p. 490-491).

C'est à la chair de la femme que Dom Polycarpe réserve sa plus intense répulsion : « ces pommes de Gomorrhe vermeilles en apparence, mais pourries au-dedans, ces parois reblanchies, mais qui crèvent de toutes parts, quels artifices, quelles sauces ne font-elles pour relever le fade goust de leur chair ?» (liv. III, chap. 6, p. 491). Les femmes sont comme certaines fleurs : «Il n'est rien si net et si blanc que le lys, l'agencement de cete fleur est fort propre, le satin fort doux et poly, ces languettes dorées qui decorent le dedans sont fort gentillement appropriées ; mais tousjours l'odeur n'en est pas seulement insuave, ains tres-puante et mauvaise ».

Ce dégoût ressenti pour la chair de la femme, la répulsion qu'il exprime, sont les symptômes des contradictions d'un désir d'amour vécu au plus profond du moi. Un passage du Livre II est de ce point de vue le révélateur. Dans l'iconographie de l'époque, le satyre est l'image du désir débridé, la chèvre et le bouc sont les deux figures, mâle et femelle, de la lubricité ${ }^{35}$. Dom Polycarpe détourne la figure de la chimère que Bellérophon, dans le mythe, tue par ordre du roi Proétos, pour expier le désir qu'Antéia, la femme de Proétos, a manifesté son égard. La Chimère

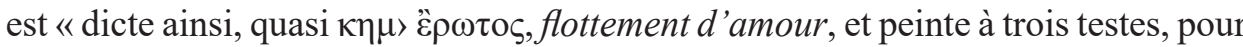
les trois degrez qu'il [l'acte sexuel] a, l'entrée, la jouissance et la fin. Car quand il commence à naistre, il assaut et donne furieusement en Lion [...] La chèvre qui est au milieu, est l'accomplissement du désir charnel [...] Finalement ce qu'on assigne à la Chimère, le derrière de Serpent, c'est parce qu'après cette satisfaction sensuelle, le venin du péché se descouvre [...] » (liv. II, chap. 1, p. 194-195).

Juste après ce passage, Dom Polycarpe semble se reprendre : certes, Socrate

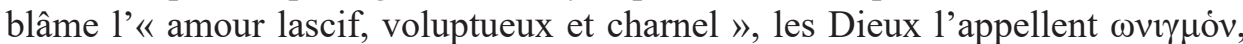
« c'est-à-dire l'estouffement du vray amour », mais Proclus interprète différemment ce passage de Platon : « cette lucte et compression est l'amour divin qui tire les âmes hors des corps corruptibles et caduques pour les enlever là-haut au ciel avec luy » (p. 195). C'est, écrit-il, ce que Salomon appelle la « Sapience ». L'amour conduit l'homme, ce vrai miracle de la nature, à une «condition toute divine », comme l'a proclamé Hermès. Dom Polycarpe cite à ce sujet, en manchette, les chapitres 3 et 4 du livre XXIII des Lectionum Antiquarum Libri XXX de Ludouicus Cælius Rhodiginus (1469-1525), ainsi que le Mercurii liber de Voluntate Dei d'Asclepius,

${ }^{35}$ Voir à titre d'exemple A. Alciat, op. cit., fo $102 \mathrm{r}^{\mathrm{o}}-\mathrm{v}^{\mathrm{o}}$ : emblema LXXII Luxuria ; fo 106 ro $107 \mathrm{r}^{\mathrm{o}}$ : emblema LXXV In amatores meretricum; fo $134 \mathrm{r}^{\circ}-135 \mathrm{v}^{0}$ : emblema XCVII Natura. 
d'après l'Exercitatio 287 de Scaliger. On reconnaît dans ce qu'il écrit l'écho de ses lectures de Ficin, dans le milieu de la Cour de Marguerite de Valois ${ }^{36}$.

Ce n'est que plus loin, dans un des chapitres suivants (chap. V, p. 234), que Dom Polycarpe cite le sermon 48, In Canticum de Bernard de Clairvaux, une des sources de la spiritualité cartusienne ${ }^{37}$. L'amour de Dieu, chez Saint Bernard, est un feu qui embrase le cœur. C'est à cet amour que l'âme de Polycarpe aspire : "Dilectus meus, dira ma chère âme, candidue et rubicundus » (p. 234 ${ }^{38}$. L'opposition entre ces deux sources que cite Dom Polycarpe, en révèle une autre plus profonde et personnelle : Le feu du désir charnel brûle toujours dans Dom Polycarpe, alors même qu'il aspire à connaître le feu de l'amour divin.

De ce désir, la dénonciation des «plus voluptueuses charnalitez » de la femme, au chapitre suivant (Liv. II, chap. XIII, p. 386) est en fait l'aveu. Dom Polycarpe y évoque avec un délice à peine contenu, « une face doucement arrondie, sa tresse blonde naturellement ondée [...]; un front argenté dévalant doucement et se tournant mollement vers les temples, les beaux yeux gros et noirs comme ébène, les joues vermeilles et potelées, les lèvres pourprines, la rangée des dents, perles menues et luisantes » (p. 250). Il ne fait guère de doute que le monde de la chair pour lequel Dom Polycarpe déclare un mépris teinté de dégoût, a été le lieu où il a aussi connu de délicieux désordres de l'âme. La sensualité, pour le choriste qu'il fut, est comme une musique de l'âme ${ }^{39}$. Dans son cas, l'appel de la dilectio bernardine se révèle impuissant à transcender l'immanence de l'amour-désir" ${ }^{40}$.

Chez Dom Polycape, le mépris du monde est l'expression de l'adieu qu'il tente de dire aux simulacres du Monde qui hantent son moi. Presqu'un siècle plus tard, Spinoza dans son Éthique tentera d'appliquer la voie démonstrative à une analyse des affects du moi. Au livre III de l'ouvrage («Proposition $15 »$, « Corollaire »

${ }^{36}$ Sur le rôle de Proclus dans l'interprétation par Marcile Ficin de la notion d'amour chez Platon et Plotin, voir P. Hadot, " L'Amour magicien'. Aux origines de la notion de magia naturalis : Platon, Plotin, Marcile Ficin », Revue Philosophique De La France Et De L'Étranger, 1982, vol. $172, n^{\circ} 2$, p. 283-292.

${ }^{37}$ Le catalogue de la bibliothèque de la Grande Chartreuse cité note onze textes du « Doctor Mellifluus », compris les Sermones sancti Bernardi super Cantica.

${ }^{38}$ En manchette : "Hebræi habent, uexillatus ex myriadibus apud nero 70 [la version des Septante].

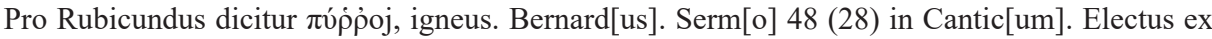
millibus quia millia millium dilecto et decies centena millia circa dilectum, et nemo ad dilectum".

${ }^{39}$ Dans un passage étonnant inspiré de Ponthus de Thyard, il écrit « ce n'est pas que Dieu n'aille quelquefois se promenant par les espaces des sens, par l'ouïe, quand sur l'harmonie musicale des voix et instrumens, il s'imagine les beaux concerts des anges ès cieux; quand sur les saveurs corporelles, on ratiocinne sur les spirituelles du banquet de l'Agneau; du toucher, quand de l'égale poli[s]sure du corps on passe au contentement de l'âme, disant avec la servente amoureuse tenui eum nec dimittam », (p. 587).

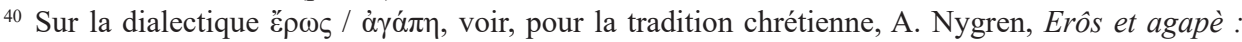
la notion chrétienne de l'amour et ses transformations, Paris, Éditions du Cerf, 2009, nouv. éd., 3 vol. En littérature française, voir P. Gifford, Love, Desire and Transcendance in French Literature, Aldershot, Ashgate, 2005. 
et « Proposition 27 »), le philosophe écrit que le plaisir que l'on prend à un objet ou la détestation que l'on ressent à son égard sont deux déterminations qui naissent de l'étonnement. Spinoza, raisonne more geometrico. Il voit dans ces deux déterminations, deux affects opposés. Mais amor et odium ne sont que les deux affects qui naissent du désir dans l'homme. Les simulacres du désir charnel ont hanté Dom Polycarpe dans sa solitude cartusienne et continuent de le faire dans l'Adieu du Monde. Il écrit pour les exorciser " plustôt pour un soulagement d'esprit, et pour éviter qu'emmy [sic] le doux repos de [s]es solitaires pensees, ce singe des actions humaines, ce Morphée seul d'entre les songes, qui sçait le mieux imiter la façon, le port et la parole de ceux qu'il représente, ne vint s'appuyer sur le lict de [s]a tranquillité », reconnaît-t-il dans $l^{\prime}$ « Advertissement » $\left(\mathrm{f}^{\circ} 2 \mathrm{r}^{\circ}-\mathrm{v}^{\circ}\right)$.

Dom Polycarpe parle de sa conversion, comme d'une " résolution », comme du choix délibéré qu'il a fait de ne plus « dresser l'oreille » au « bruit inopiné qui tabute » son âme, (liv. IV, chap. IV p. 723). Saint Paul et Saint Augustin sont les deux prototypes de la conversion chrétienne. Saint Paul (Actes des Apôtres, 22. 6-7) est terrassé par une lumière soudaine. À l'opposé, celle de Saint Augustin est le résultat d'un combat toujours présent dans son âme entre caritas et cupiditas. Comme l'évêque d'Hippone en fait lui-même l'aveu au livre X chapitre XXXIV, des Confessions, «l'illusion de l'image a tant de force dans mon âme sur ma chair, que ces visions irréelles obtiennent de moi pendant le sommeil ce que la vision des réalités ne peut obtenir quand je suis éveillé $»^{41}$. Dom Polycarpe cite Saint Augustin au moins dix-sept fois dans L'Adieu du Monde mais on ne découvre, dans l'ouvrage, ni le sentiment de libération qui suit l'aveu chez Saint Augustin, ni l'illumination paulinienne.

Il serait erroné d'y voir un manque d'authenticité et de réduire ce texte à des variations savantes sur un lieu commun. Dom Polycarpe ne disposait d'autre langage pour parler de lui-même que celui que lui avaient fourni ses lectures. Son univers mental s'était formé dans l'entourage de la Cour de Marguerite de Valois exilée dans son château d'Usson, où il avait vécu jusqu'à l'âge adulte, Il restait tributaire d'une culture qu'il y avait acquise, faite d'un culte des Anciens teinté de platonisme chrétien.

Dans cette culture, la citation et la paraphrase étaient des formes courantes d'écriture, et dans ce milieu le mépris du Monde était une posture, une forme de défi aux vicissitudes de l'exil imposé. Le silence de la Grande Chartreuse l'a mis face à lui-même et déstabilisé les certitudes qu'il avait acquises. Comme Thomas More, l'auteur de l'Utopie (1516), qui lui aussi séjourna quatre ans durant dans une Chartreuse, François de la Rivière rentra dans le Monde aux prises avec un sentiment d'échec religieux et moral. La citation-répétition fonctionne dans Le Mépris du

${ }^{41}$ Livre X, XXX 41, "et tantum ualet imaginis illius inlusio in anima mea in carne mea" (version française de É. Tréhorel et G. Bouissou, Euvres de saint Augustin, 2e éd., Paris, Études augustiniennes, 1998. 
Monde comme un « transfert imaginaire » lacanien. Dans la rhétorique du discours, citations, arguties logiques et philologiques sont les instruments inconscients d'une stratégie de l'offuscation, par déplacement et condensation ${ }^{42}$. Ce qui distingue L'Adieu du Monde et qui confère à l'œuvre sa singularité pour l'époque, est la façon dont, dans son écriture, la delectatio morosa à la fois voile et dévoile ce qu'elle ne peut avouer.

\section{Bibliographie}

\section{Sitographie}

Dictionnaire de spiritualité ascétique et mystique, doctrine et histoire, Paris, Beauchesne ; URL : http:// beauchesne.immanens.com/appli/article.php?id=12457 ; consulté le 2.11.2018

Nouvelle biographie cartusienne, édition revue, corrigée et augmentée, $2^{\mathrm{e}}$ partie, Grande Chartreuse, 2005, l'article « De la Rivière, Polycarpe », vue 110 ; URL : https://cercornum.univ-st-etienne.fr/ analectacartusiana/sites/default/files/acpdfsbillets/NBC_2-Religieux_chartreux-2005.pdf; consulté le 2.11.2018

Daronnat, Éric, « Lexique mystique. France - XVII siècle », mis à jour en 2014 ; URL : https://grac. univ-lyon2.fr/lexique-mystique-1-r--565754.kjsp ; consulté le 2.11.2018

\section{Bibliographie primaire}

Alciat, André, Emblemata Andreae Alciati I. C. clariss. Latinogallica / Les Emblemes latin-françois du seigneur André Alciat excellent jurisconsulte, trad. Claude Mignault, Paris, Jean Richer, 1584

Desportes, Philipe, Les Premieres œuvres de Philippes Des Portes, Au Roy de Pologne, Paris, Robert Estienne, 1573

De La Roche Chandieu, Antoine, Méditations sur le psalme XXXII, trad. du latin en Français ont été adoustez 50 octonaires sur la vanité du monde par Antoine de Zamariel, Genève, G. Laimaire, 1583

L'Armorial historique de Bresse, Bugey, Dombes, Pays de Gex, Valromey et Franc-Lyonnais, éd. le Révérend Édmond Du Mesnil, Lyon, 1872

Dom Polycarpe de la Rivière, Recreations Spirituelles sur l'amour divin et le bien des amis. Enrichies d'une infinité d'Inventions tres-subtiles et utiles à la Conversion des grandes Ames de la Cour. Tirées de la Bibliotheque de Mre Baltazar de Villars, Conseiller du Roy en son Conseil d'Estat, President en la Seneschaulcée et Siege Presidial de Lyon et premier President au Parlement de Dombes, Paris, chez Regnauld Chaudiere, 1617

Dom Polycarpe de la Rivière, L'Adieu du monde, ou Le mépris de ses vaines grandeurs et plaisirs périssables, Antoine Pillehotte, Lyon, 1619

De Roa, Martin, Singularium locorum ac rerum libri V, Lugduni, sumptibus Horatii Cardon, 1604

Fournier, Paul, Notice sur la bibliothèque de la Grande Chartreuse au Moyen Âge. Suivie d'un Catalogue de cette bibliothèque au XV siècle, Grenoble, Allier, 1888

Julii Caesaris Scaligeri Exotericarum exercitationum liber quintus decimus, de subtilitate, ad Hieronymum Cardanum, Lutetiæ, Michaelis Vascosiani, 1557

Les Dialogues de Jean Loys Vives, traduits du latin en françoys [...] par Gilles de Housteville, Anvers, Guillaume Cuzman, 1578

Migne, Jacques, Paul, Patrologia Graeca, t. LXIII ; URL : http://patristica.net/graeca/ ; consulté le 15.09.2019

${ }^{42}$ J. Lacan, Les Quatre concepts fondamentaux de la psychanalyse : 1964 ; texte établi par J.-A. Miller, Paris, Seuil, 1992. 
Bibliographie secondaire

Actes du Colloque sur l'humanisme lyonnais au XVI' siècle. Mai 1972, Grenoble, Presses Universitaires de Grenoble, 1974

Actes du Colloque de Nérac, De Marguerite de Valois à la reine Margot autrice, mécène, inspiratrice, sous la dir. de Catherine Magnien, Éliane Viennot, Rennes, Presses Universitaires de Rennes, 2019

Chaix, Gérald, Réforme et contre-réforme catholiques. Recherches sur la chartreuse de Cologne au $X V I^{e}$ siècle, Salzburg (Analecta cartusiana 80), 1981, t. 3

Chaix, Gérald, «Contributions cartusiennes aux débuts de la réforme catholique dans les pays de langue française (1560-1620)», Revue d'histoire de l'Église de France, 1989, nº 194, p. 115-123 https:// doi.org/10.3406/rhef.1989.3459

Conversion au XVIIe siècle [La], Actes du XII colloque de Marseille, janvier 1982, éd. Louise Godard de Donville, Marseille, Centre méridional de rencontres sur le XVII ${ }^{\mathrm{e}}$ siècle, 1983

Gifford, Paul, Love, Desire and Transcendence in French Literature, Aldershot, Ashgate, 2005

Intellectual Life in Renaissance Lyon. Proceedings of the Cambridge Lyon colloquium, 14-16 April 1991, ed. by Philip Ford, Gillian Jondorf, Cambridge, Cambridge French Colloquia, 1993

Kappler, Émile, Les Conférences théologiques entre catholiques et protestants en France au XVII siècle, Paris, Honoré Champion, 2011

Lacan, Jacques Les Quatre concepts fondamentaux de la psychanalyse: 1964; [texte établi par JacquesAlain Miller], Paris, Seuil, 1992

Mariéjol, Jean-Hippolite, La Vie de Marguerite de Valois : reine de Navarre et de France : 1553-1615, Paris, Hachette, 1928

Meylan, Édouard F., «L'évolution de la notion d'amour platonique », Humanisme et Renaissance, 1938 , t. $5, n^{\circ} 3$, p. $418-442$

Moss, Ann, Les Recueils de lieux communs, méthode pour apprendre à penser à la Renaissance, traduit de l'anglais sous la dir. de Patricia Eichel-Lojkine, Genève, Droz, 2002

Nygren, Anders, Erôs et agapè : la notion chrétienne de l'amour et ses transformations, Paris, Éditions du Cerf, 2009, nouv. éd., 3 vol.

Viennot, Éliane, Marguerite de Valois : «la reine Margot », Paris, Perrin, 2005

Jean-Paul Pittion est ancien élève de l'École Normale Supérieure, Docteur de l'Université de Dublin et Fellow emeritus du Trinity College de Dublin. Il a été Professeur au Centre d'Études Supérieures de la Renaissance de Tours. Sa thèse de doctorat ( $\mathrm{Ph}$. D.) portait sur la vie intellectuelle de l'Académie protestante de Saumur au dix-septième siècle. Ses publications récentes incluent Le Livre à la Renaissance : introduction à la bibliographie historique et matérielle (Brepols, 2014).

\begin{tabular}{|l|l|}
\hline CC) Compative & $\begin{array}{l}\text { C by the author, licensee Łódź University - Łódź University Press, } \\
\text { Łódź, Poland. This article is an open access article distributed under } \\
\text { the terms and conditions of the Creative Commons Attribution license } \\
\text { CC-BY-NC-ND 4.0 (https://creativecommons.org/licenses/by-nc-nd/4.0/) }\end{array}$ \\
\cline { 2 - 2 } & Received: 2019-01-20; Accepted: 2020-11-03 \\
\hline
\end{tabular}

